\title{
Características clínicas de la enfermedad respiratoria causada por Mycoplasma pneumoniae en niños hospitalizados
}

\author{
M. de los Ángeles Paul D., Luis E. Vega-Briceño, Marcela Potin S., Marcela Ferrés G., \\ Dahiana Pulgar B., Cristián García B., Linus Holmgren P. e lgnacio Sánchez D.
}

\section{Clinical characterizes of respiratory infection due to Mycoplasma pneumoniae in hospitalized children}

Introduction: Studies on Mycoplasma pneumoniae infection are scarce in Chile. Objective: To describe clinical characteristics associated with M. pneumoniae in children requiring hospitalization. Material and Methods: All children with a respiratory infection requiring hospitalizations between 2000-2005, whom had a M. pneumoniae specific IgM $\geq 1: 32$, were analyzed. Results: Fifty children meeting study criteria were identified with an average length of hospitalization of 4 days (range: 1-10); mean age was 5.4 years (46\% were younger than 5 years). Common clinical features were cough (92\%), fever (82\%), malaise (74\%) and respiratory distress (72\%). At admission 40/45 children had hypoxemia. Chest-X ray showed interstitial pattern $(69.3 \%)$, consolidation (51\%) and hyperinsuflation (28.5\%). Six patients had pleural effusion. Eighty four percent of patients had a favorable clinical outcome; eight children required admission to the PICU all of whom recovered. Conclusion: Respiratory infections associated with M. pneumoniae in our series of children had a highly variable and non-specific clinical spectrum. Chest-X rays showed different pattern in concordance with previous publications.

Key words: Mycoplasma pneumoniae, complication, hospitalization, pneumonia, children.

Palabras clave: Mycoplasma pneumoniae, complicaciones, hospitalización, neumonía, niños.

\section{Introducción}

$\mathrm{E}$ 1 género Mycoplasma representa los microorganismos auto-replicativos más pequeños descritos por el hombre, y comprende a un grupo de bacterias carentes de pared celular, responsables de diversas infecciones en el ser humano ${ }^{1}$. Desde mediados del siglo XX, M. pneumoniae es reconocido como agente etiológico de cuadros de neumonía atípica ${ }^{2}$, que afecta tanto a niños como adultos en forma endémica, presentando brotes preferentemente durante verano y principios de otoño ${ }^{3-6}$ aproximadamente cada cuatro años ${ }^{3}$.

Actualmente, $M$. pneumoniae es responsable de hasta $\sim 40 \%$ de las neumonías adquiridas en la comunidad (NAC) en niños ${ }^{7-9}$ y $\sim 18 \%$ de las NAC que requieren hospitalización $^{10,11}$. Se estima que, del número total de NAC causadas por M. pneumoniae, sólo 2 a $4 \%$ requerirá hospitalización ${ }^{12}$. Un estudio en nuestro medio ${ }^{13}$, investigó la presencia de $M$. pneumoniae en secreción faríngea de 185 niños asintomáticos, detectando $2 \%$ de portación faríngea. No existen datos locales que revelen cuál es la prevalencia de esta infección en niños.

Clásicamente, se ha afirmado que la infección por M. pneumoniae es poco frecuente bajo 5 años de edad, describiéndose como grupo de mayor riesgo los niños escolares $^{14-17}$; con disminución considerable hacia la adolescencia y edad adulta ${ }^{18}$. El cuadro clínico se caracteriza por un inicio gradual, con cefalea, malestar general y fiebre; son comunes la faringitis, bronquitis y menos frecuente la otitis media aguda ${ }^{17,19}$. En general, las manifestaciones clínicas son inespecíficas, siendo el cuadro usualmente benigno ${ }^{18} \mathrm{y}$ autolimitado ${ }^{16,20,21}$. El objetivo de este estudio fue describir el perfil clínico y radiológico de los pacientes hospitalizados con enfermedad causada por M. pneumoniae en el Servicio de Pediatría de la Pontificia Universidad Católica (PUC) de Chile.

\section{Pacientes y Métodos}

Se revisó la base de datos del Servicio de Pediatría del Hospital Clínico de la PUC identificando todas las hospitalizaciones, por alguna causa respiratoria, durante el período 2000-2005. Se consultó el resultado de los exámenes de laboratorio de cada paciente, en forma electrónica, identificando a todos aquellos con títulos positivos de IgM para M. pneumoniae por técnica de inmunofluorescencia (Mycoplasma pneumoniae IgM IFA ${ }^{\circledR}$, Zeus Scientific, Inc.). Se consideró como resultado positivo la presencia de fluorescencia verde manzana 2 a 3 positivo (en títulos $\geq 1: 32$ diluciones) en la muestra observada. De acuerdo
Pontificia Universidad Católica de Chile, Santiago Escuela de Medicina (MAPD, DPB) Departamento de Pediatría (LEVB, MPS, MFG, LHP, ISD)

Departamento de Radiología (CGB)

Recibido: 17 de diciembre de 2008 Aceptado: 4 de abril de 2009

Correspondencia a: Luis Enrique Vega-Briceño levega@puc.cl 
a las especificaciones del fabricante, la sensibilidad y especificidad de la prueba son de 89 y $95 \%$, respectivamente. La presencia de factor reumatoideo (FR) puede ser causa de falsos positivos; la técnica usada no incluía en ese momento la captura previa de FR.

Luego se revisó la ficha médica de cada niño, registrando antecedentes mórbidos de importancia, síntomas y signos que motivaron la consulta, hallazgos en el examen físico y parámetros de laboratorio al ingreso y complicaciones durante la hospitalización.

Definiciones. Hipoxemia: saturación arterial de oxígeno transcutánea $\leq 90 \%$. Alguna condición de base: antecedentes de prematurez, alergia, sibilancias recurrentes, genopatía, patología cardíaca o respiratoria crónica, enfermedad neurológica, inmunodeficiencias o malignidades hematológicas. Sibilancias recurrentes: presencia

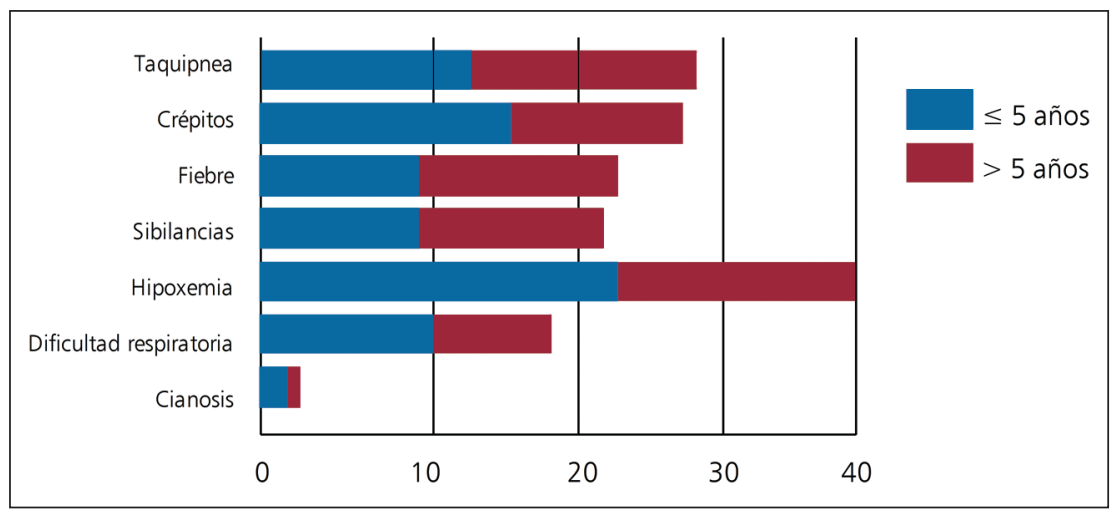

Figura 1. Examen físico al ingreso. Frecuencia (n) de hallazgos.

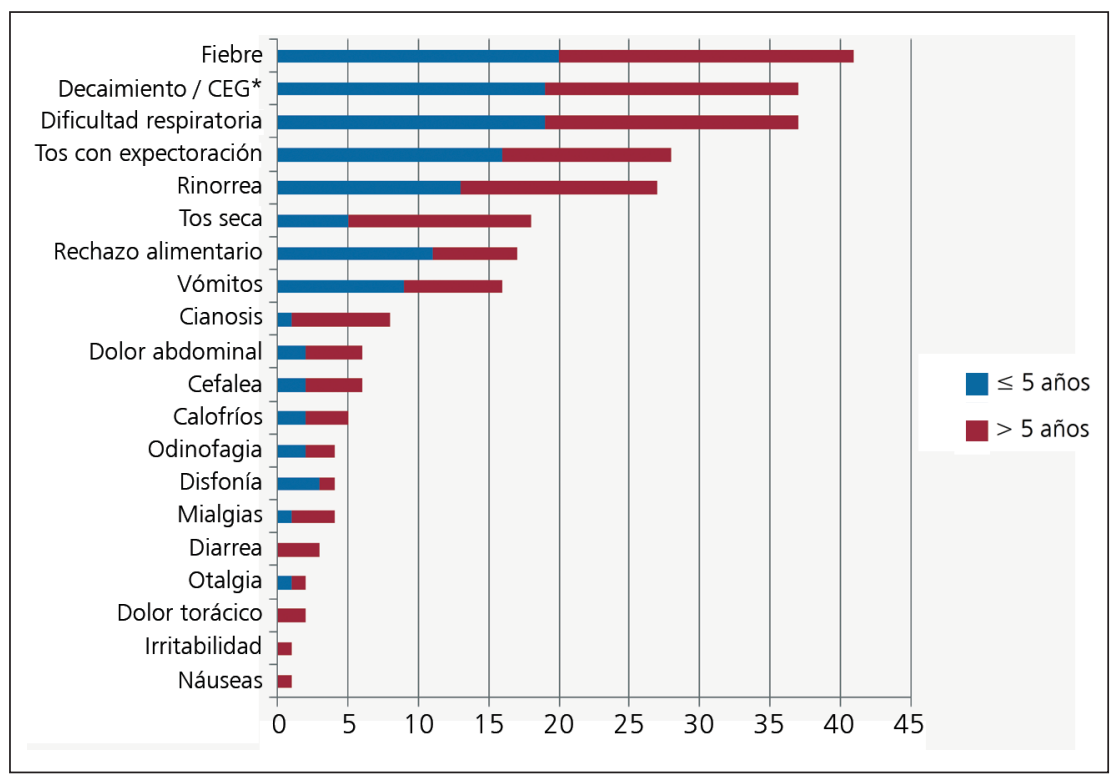

Figura 2. Síntomas previos al ingreso. Frecuencia (n) de hallazgos. * CEG: Compromiso del estado general. de $\geq 3$ episodios de sibilancias, confirmadas por médico. Sibilancias asociadas a virus: conjunto bronquiolitis (bajo un año de edad), bronquitis obstructiva o crisis asmáticas.

Análisis estadístico. Se empleó la mediana como medida de tendencia central y rangos para establecer la dispersión de la muestra. Las frecuencias fueron reportadas como frecuencias simples. Se generó una hoja Excel y se empleó el programa STATCALC versión 5,4 para el análisis estadístico.

\section{Resultados}

Durante el período seleccionado se internaron 4.356 niños por alguna causa respiratoria. De éstos, se identificaron $65(1,5 \%)$ pacientes con IgM positiva para M. pneumoniae, lográndose revisar las fichas médicas de 46 niños con un total de 50 hospitalizaciones o eventos. Del total de pacientes, 21/46 eran hombres. La edad promedio fue 5,4 años (rango: 15 meses - 12,4 años). Veintitrés (46\%) internaciones ocurrieron en pacientes bajo 5 años de edad. Cuarenta y dos $(65 \%)$ hospitalizaciones ocurrieron entre junio y noviembre. Dos niños estuvieron internados dos veces en el período y uno tuvo tres hospitalizaciones.

Con respecto a antecedentes mórbidos, 40 tenían antecedente de sibilancias alguna vez (27 con sibilancias recurrentes) y 16 neumonía. Ocho niños habían sido hospitalizados por alguna causa respiratoria (tres por $M$. pneumoniae) durante los 12 meses previos al ingreso actual. En 7/50 niños existía el antecedente de genopatía o enfermedad neurológica, cuatro de ellos correspondían a síndrome de Down (dos con cardiopatía asociada) y los otros a neurofibromatosis, parálisis del plexo braquial y leucomalacia periventricular, respectivamente.

Se logró rescatar los datos del servicio de urgencia (SU) en 45/50 casos. En 23 de ellos se constató fiebre, en 28 taquipnea, en 27 crépitos, en 22 sibilancias y en 18 dificultad respiratoria. Dos presentaron cianosis y dos mostraron una otoscopia compatible con otitis media aguda. En el SU 40/45 pacientes presentaron hipoxemia. En la Figura 1 se observa el examen físico según edad del paciente. Los signos y síntomas que motivaron la consulta se observan en la Figura 2. El promedio de duración de los síntomas fue 7 días (rango: 1-45). Ochenta y cuatro por ciento de los pacientes habían consultado previamente, con un promedio de consultas de 1,4 (rango: 1-4). En $82 \%$ se documentó fiebre, con una duración de 2,7 días (rango: 1-7 días). Finalmente, el tiempo promedio de hospitalización fue de cuatro días (rango: 1-10), siendo en 17/65 casos la hospitalización menor a cuatro días. En $54 \%$ se había utilizado algún antimicrobiano previamente (macrólidos en cinco casos).

En 98\% de los casos se tuvo acceso a la descripción radiológica (Tabla 1 y Figuras 3-5): infiltrado intersticial 
(n: 34), foco de consolidación (n: 25), hiperinsuflación (n: 14), atelectasia (n: 10) y efusión pleural (n: 6). Ningún paciente presentó anemia (definido como hematocrito menor a 2 DS del valor normal para la edad y sexo, según valores de referencia del laboratorio clínico de nuestra institución) y 10/41 tenían leucocitosis $\left(>15.000 / \mathrm{mm}^{3}\right)$, 16/41 de ellos con desviación a izquierda. En 22/34 casos la PCR fue mayor a $3 \mathrm{mg} / \mathrm{dl}$ y $31 / 37$ tenían VHS elevada (mayor a $24 \mathrm{~mm} / \mathrm{h}$ en mujeres y $14 \mathrm{~mm} / \mathrm{h}$ en hombres). Se demostró co-infección en tres sujetos: virus influenza

Tabla 1. Hallazgos radiografía de tórax en neumonía adquirida en la comunidad asociada a Mycoplasma pneumoniae, según distintas series publicadas

\begin{tabular}{|c|c|c|c|c|c|c|}
\hline \multirow[b]{2}{*}{ Alteraciones radiológicas } & \multicolumn{6}{|c|}{ Porcentaje de casos } \\
\hline & $\begin{array}{c}\text { Hospital } \\
\text { UC }\end{array}$ & John y cols. ${ }^{26}$ & Esposito y cols. ${ }^{31}$ & Othman y cols. ${ }^{28}$ & Shenoy y cols. ${ }^{41}$ & Reittner y cols. ${ }^{\# 42}$ \\
\hline Normal & 2 & 5 & & 2,8 & 31,8 & 11 \\
\hline Patrón intersticial difuso & 69,4 & 7 & & 30,5 & & \\
\hline Consolidación & 51 & 33 & $27,9^{*} / 7,4^{* *}$ & 16,7 & 4,5 & 86 \\
\hline Hiperinsuflación & 28,6 & & 14,7 & 16,7 & & \\
\hline Atelectasias & 20,4 & 29 & & & & \\
\hline Efusión pleural & 12,2 & 17 & 5,9 & 8,3 & 4,5 & 7 \\
\hline Patrón reticular focal & & $52 * / 10 * *$ & & & & \\
\hline Engrosamiento broncovascular & & 12 & 4,4 & & & 18 \\
\hline Adenopatías & & 7 & & 1,4 & & 10 \\
\hline Infiltrado retículo-nodular & & & 39,7 & & & \\
\hline Opacidades lineales & & & 60,3 & & & 10 \\
\hline Nódulos & & & & & & 50 \\
\hline Opacidades bilaterales & & & & 22,9 & 18,2 & \\
\hline Opacidades perihiliares & & & & & 4,5 & \\
\hline
\end{tabular}
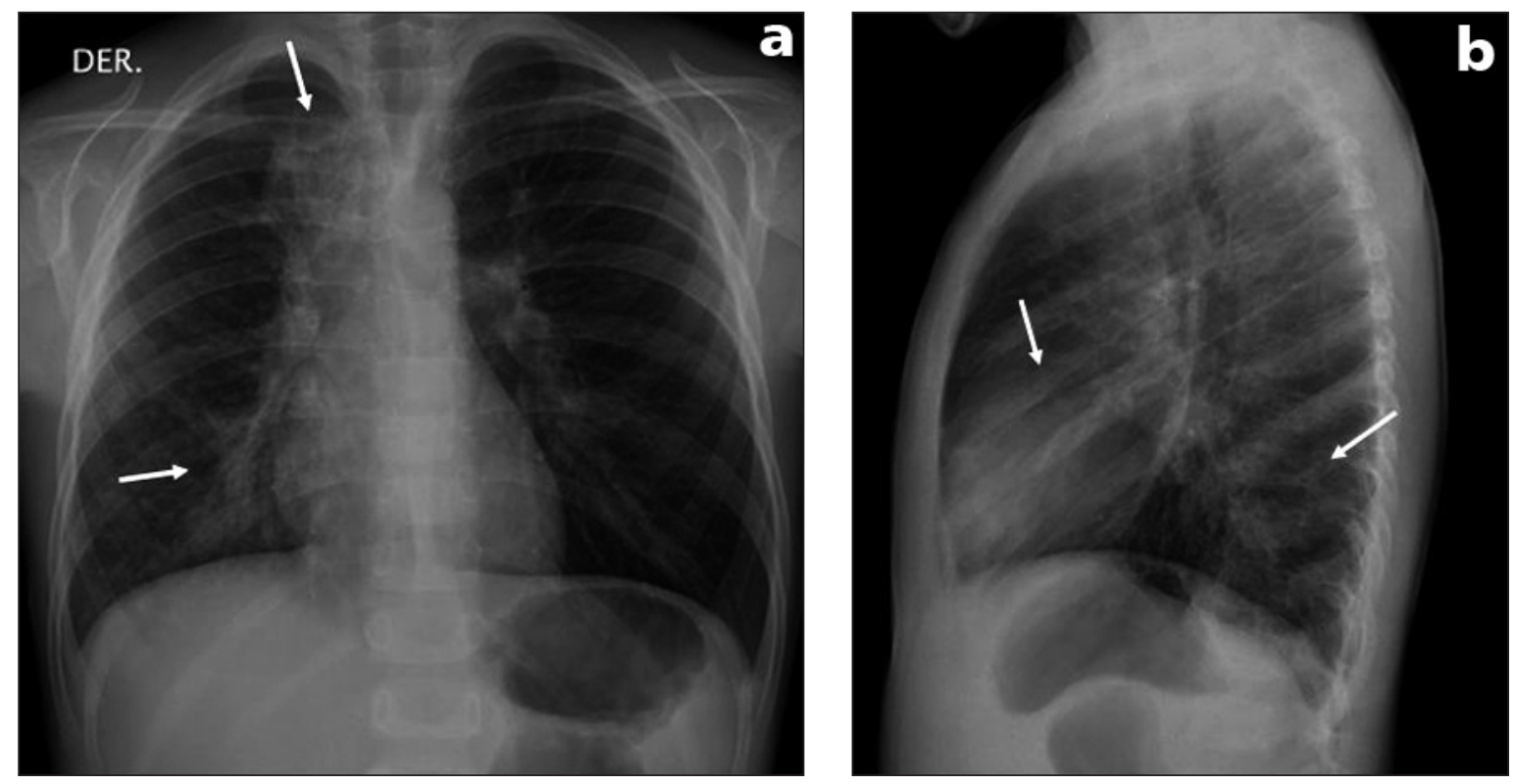

Figura 3. Rx de tórax PA (a) y lateral (b), muestran sombras parcialmente confluentes en los lóbulos superior y medio derechos (flechas), con un componente atelectásico. 
Figura 4. Rx de tórax PA (a) y lateral (b), muestran sombras de predominio intersticial, con algunas áreas de compromiso alveolar, en ambos lóbulos inferiores y en el lóbulo medio derecho (flechas).

Figura 5. Rx de tórax PA (a) y lateral (b), muestran sombras parcialmente confluentes en los lóbulos superior y medio derechos (flechas), con un componente atelectásico.
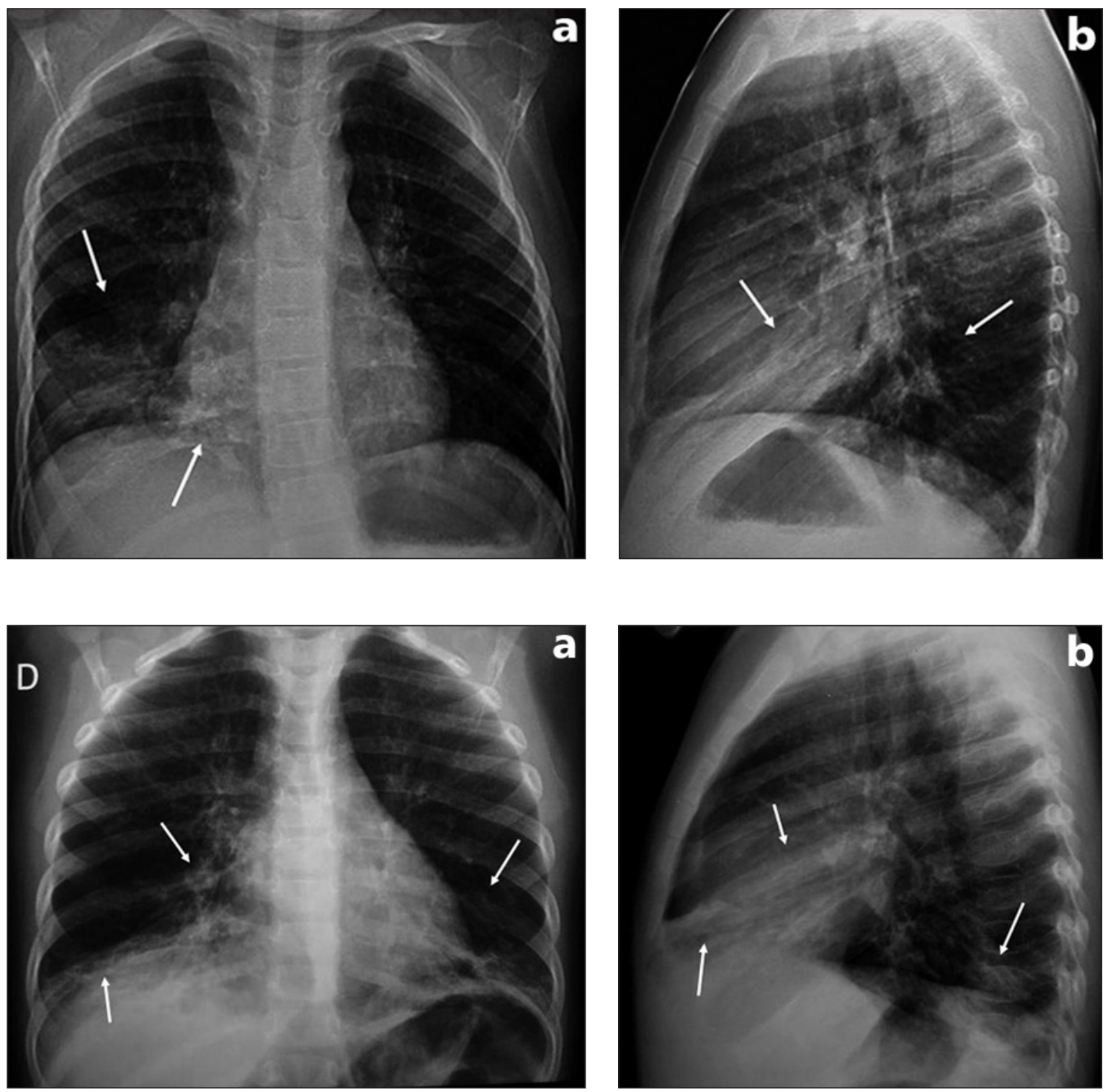

A (n: 1), Bordetella pertussis (n: 1) y virus respiratorio sincicial y $B$. pertussis (n:1), simultáneamente. En $84 \%$ la evolución fue favorable, cursando toda su hospitalización en sala. Tres pacientes evolucionaron con exantema y uno presentó una convulsión febril durante la evolución. Ocho pacientes estuvieron hospitalizados en la Unidad de Paciente Crítico, requiriendo cuatro de ellos ventilación no invasora (VNI). No se registraron fallecidos.

\section{Discusión}

Este es el primer reporte publicado en nuestro medio que describe el perfil clínico-radiológico de un grupo reducido de pacientes pediátricos hospitalizados por infección asociada a M. pneumoniae. Pese a las limitaciones de una descripción retrospectiva (inherentes a un registro incompleto), casi la mitad de las hospitalizaciones ocurrieron en niños bajo 5 años de edad, similar a lo descrito por algunos investigadores ${ }^{7,8,22,23}$, y diferente a lo descrito de manera clásica o tradicional ${ }^{14-17}$.

Mycoplasma pneumoniae es causa frecuente de infecciones respiratorias en niños ${ }^{10}$. En población pediátrica europea y norteamericana causa hasta $40 \%$ de las NAC en este grupo etario ${ }^{7-9}$. Según Ferrero y cols, hasta $15 \%$ de las NAC que requieren hospitalización en niños argentinos son secundarias a M. pneumoniae ${ }^{24}, \mathrm{y}$ en un estudio realizado en Panamá en niños con NAC 
ambulatoria se observó una prevalencia de $7 \%$ de $M$. pneumoniae $^{25}$. En Chile no se conoce la prevalencia de esta enfermedad.

La infección por $M$. pneumoniae puede ser asintomática, en $\sim 20 \%$ de los casos, o puede afectar la vía aérea superior o inferior o ambas, apareciendo síntomas que se desarrollan en un período de varios días y que pueden persistir por semanas o meses ${ }^{10}$. En nuestra serie clínica, el promedio de duración de los síntomas previo al ingreso fue 7 días, prolongándose hasta 45 días en un caso (quien mejoró luego de iniciada la terapia con macrólidos); casi la mitad tuvo una evolución mayor a 7 días. John et $\mathrm{al}^{26}$, describe como cuadro sospechoso de enfermedad por $M$. pneumoniae aquel que dura más de una semana, caracterizado por tos seca persistente, fiebre baja (menor a $38,5^{\circ} \mathrm{C}$ ) o ausencia de fiebre asociado a cefalea o mialgias, siendo la rinorrea y/o congestión nasal infrecuentes. La tos se describe como inicialmente seca pero luego presenta expectoración escasa. Braun y $\operatorname{cols}^{27}$, describen la enfermedad por M. pneumoniae como influenza-like, y sugieren sospecharla en pacientes con tos con expectoración, fiebre, cefalea y mialgias. En nuestra serie, los síntomas referidos con mayor frecuencia fueron fiebre, compromiso del estado general y tos, similar a lo descrito por Waites ${ }^{10}$. En la mayoría de los casos, la tos era productiva. Destaca además una alta prevalencia de rinorrea, lo que previamente ha sido descrito como infrecuente en la enfermedad por M. pneumoniae ${ }^{16,17}$. Síntomas como cefalea y/o mialgias se observaron sólo en alrededor de $10 \%$ de los casos.

Con respecto al examen físico, en la mitad de los casos de esta serie se consignaron sibilancias. Othman y cols ${ }^{28}$, describe que en más de un tercio de las NAC causadas por M. pneumoniae en niños hospitalizados se encontrarían sibilancias. La alta prevalencia de sibilancias en nuestra experiencia se acompaña de una alta frecuencia de historia previa de sibilancias o asma, lo que es compatible con el rol asignado a $M$. pneumoniae como gatillante o exacerbador de asma en niños y adultos ${ }^{29,30}$. Por otro lado, destaca la presencia de crépitos en alrededor de dos tercios de los casos, cifra menor a lo descrito previamente por Esposito y cols ${ }^{31}$ y Principi y cols ${ }^{32}$.

Las manifestaciones clínicas varían según la edad del paciente, se ha descrito que bajo 5 años de edad predominan habitualmente síntomas respiratorios altos y sibilancias, aumentando la prevalencia de NAC en el grupo entre los 5 y 15 años $^{3,23,33}$. En nuestra experiencia, los pacientes con sibilancias, tenían, en más de la mitad de los casos, 5 años o más (Figura 1).

Si bien la infección por M. pneumoniae es habitualmente benigna y auto-limitada ${ }^{20,21}$, en algunos casos puede producir compromiso pulmonar grave $\mathrm{e}^{34,35}$. En adultos se describe que hasta $11 \%$ de los pacientes requiere ventilación mecánica convencional ${ }^{18}$ con una mortalidad asociada de 3 a $5 \%{ }^{22}$. El riesgo de presentar una enfermedad grave aumenta en casos de infecciones concomitantes, inmunosupresión, anemia de células falciformes y síndrome de Down ${ }^{3,10}$. En nuestro estudio, de los cuatro pacientes que requirieron VNI uno presentaba síndrome de Down y otro tenía co-infección con $B$. pertussis y VRS.

Mycoplasma pneumoniae puede producir manifestaciones extra-pulmonares hasta en $25 \%$ de los casos, con complicaciones neurológicas, dermatológicas, hematológicas, cardíacas, renales y osteo-articulares ${ }^{15,36}$. En esta serie, tres pacientes evolucionaron con exantema y uno con una convulsión febril.

Para la detección de M. pneumoniae existen múltiples métodos $^{13,18,37,38}$. La serología es el método empleado con mayor frecuencia ${ }^{13}$, siendo el estándar dorado para el diagnóstico la seroconversión entre muestras de suero en fase aguda y de convalecencia ${ }^{3}$. Si bien se puede utilizar la detección de M. pneumoniae mediante cultivo o RPC a partir de muestras respiratorias, la persistencia del microorganismo por períodos variables de tiempo después de la infección aguda dificulta en algunos casos la interpretación del resultado ${ }^{39}$. En población pediátrica, un único título positivo de $\operatorname{IgM}$ en etapa aguda podría ser considerado como criterio diagnóstico ${ }^{37}$ principalmente en épocas de alta prevalencia. Existen limitaciones para establecer un diagnóstico concluyente, con una única determinación serológica. La IgM se eleva entre los 7 y 10 días de iniciado el cuadro, presentando un pico a las 4 a 6 semanas, para luego iniciar un descenso a partir de los 4 a 6 meses ${ }^{15}$. La sensibilidad de la detección de IgM varía entre 42 y $67 \%$ para la muestra en fase aguda y entre 75 y $100 \%$ al combinar las muestras de fase aguda y tardía; la especificidad varía entre $92-98 \%$ y $89-98 \%$, para uno y otro caso ${ }^{38}$. Los ensayos de inmunofluorescencia (como el utilizado en el presente estudio) son técnicamente simples de realizar, pero son operador dependiente y requieren un microscopio ad-hoc. Actualmente, los métodos más utilizados son los ensayos inmuno-enzimáticos, dada su buena sensibilidad y especificidad, y escasa cantidad de suero requerida para la prueba. Al igual que la inmunofluorescencia, las técnicas de ELISA requieren de dos muestras para confirmar el diagnóstico ${ }^{39}$, siendo una limitación en muchos ensayos clínicos. Desde 2007, la serología de $M$. pneumoniae se realiza en nuestra institución mediante una prueba de ELISA cualitativa (Zeus Scientific, Inc.). Souliu y $\operatorname{cols}^{38}$, plantean que para establecer a M. pneumoniae como etiología de un cuadro respiratorio, la IgM por método de ELISA debería ser confirmada mediante Western blot, estando asociada al menos con dos de los siguientes criterios: RPC o cultivo positivo; seroconversión de inmunoglobulinas o niveles de $\operatorname{IgG}>400 \mathrm{U} / \mathrm{ml}$. En nuestra serie no se realizó confirmación diagnóstica por otro método, dado su carácter retrospectivo.

Con respecto a los exámenes de laboratorio, $\sim$ un tercio 
de los casos presentaba leucocitosis, compatible con lo previamente descrito ${ }^{15,33}$, asociada a desviación a izquierda en un tercio de ellos. Algunos autores sostienen que los hallazgos de la radiografía de tórax pueden ser muy variados $^{16,20}$ y no permiten distinguir la neumonía por $M$. pneumoniae de la producida por otros bacterias atípicas o infecciones virales ${ }^{33,40}$. Clásicamente se ha descrito un patrón reticular intersticial difuso, siendo poco frecuentes los focos de consolidación y efusiones pleurales ${ }^{3,15-17}$. En la Tabla 1 se comparan nuestros hallazgos con lo publicado previamente en la literatura médica; destaca la presencia de consolidación en la mitad de los casos, cifra mayor a lo descrito anteriormente por John ${ }^{26}$ y Esposito ${ }^{31}$, quienes reportaron un patrón reticulo-nodular y engrosamiento peribronquial, respectivamente, con foco de consolidación sólo en un tercio de los casos. La incidencia de efusión pleural y atelectasias es similar a lo descrito con anterioridad $^{26}$. En cambio, en dos tercios de los casos la radiografía presentaba un infiltrado intersticial difuso, mayor a lo comunicado por otros autores ${ }^{26,28}$. Finalmente, en esta serie clínica se atribuyó a $M$. pneumoniae como responsable de la hospitalización; por ello, todos recibieron antibioterapia específica con macrólidos, exhibiendo una mejoría clínica sustancial.

En conclusión, la infección por M. pneumoniae en esta serie produjo manifestaciones inespecíficas y variables de un caso a otro, a veces difíciles de distinguir de otras infecciones respiratorias, incluso virales. Destacó la presencia de fiebre, tos, rinorrea y compromiso del estado general, asociados a una alta frecuencia de crépitos y sibilancias. La radiografía de torax reveló una variedad de patrones que dificultan la orientación diagnóstica sólo por imágenes. Una descripción adecuada del cuadro clínico permitirá conocer los signos y síntomas más frecuentes de presentación, lo que lleva a un mayor índice de sospecha.
Ello permitirá un inicio precoz del tratamiento antimicrobiano, acortando así la duración de la enfermedad y disminuyendo el riesgo de sus complicaciones. Debido a las limitaciones propias de una descripción retrospectiva, se sugiere complementar lo descrito anteriormente mediante nuevos estudios epidemiológicos.

\section{Resumen}

Introducción: La infección por Mycoplasma pneumoniae es una condición respiratoria poco estudiada en nuestro medio. Objetivo: Describir las características clínicas de los niños hospitalizados por M. pneumoniae. Materiales y Métodos: Se analizaron todos los pacientes hospitalizados por infecciones respiratorias durante el 2000-2005, con IgM específica; se utilizó como diagnóstico de enfermedad por $M$. pneumoniae la presencia de fluorescencia verde manzana 2 a 3 positivo en títulos $\geq 1: 32$ diluciones. Resultados: Se analizaron 50 hospitalizaciones, con estadía promedio de 4 días (rango: 1-10); la edad promedio fue 5,4 años ( $46 \%$ bajo 5 años). Los síntomas más frecuentes fueron tos $(92 \%)$, fiebre (82\%), compromiso del estado general (74\%) y dificultad respiratoria ( $72 \%$ ). Al momento del ingreso $40 / 45$ presentaron hipoxemia. La radiografía de tórax (RT) reveló infiltrado intersticial $(69,3 \%)$, foco de consolidación $(51 \%)$ e hiperinsuflación $(28,5 \%)$. Seis presentaron efusión pleural asociada. En 84\% la evolución fue favorable; sin embargo, 8 niños ingresaron a la Unidad de Paciente Critico para monitorización. No hubo decesos Conclusiones: La infección respiratoria asociada a $M$. pneumoniae en niños produjo manifestaciones inespecíficas y variables de un caso a otro. La RT reveló una variedad de presentaciones similar a lo mostrado en la literatura médica.

\section{Referencias}

1.- Baseman J, Tully J. Mycoplasmas: Sophisticated, re-emerging and burdened by their notoriety. Emerg Infect Dis 1997; 3: 21-32.

2.- Mulson M, Bloom H, Manko M, Kingston J, Chanock R. Acute respiratory diseases of viral etiology. V. Eaton agent: a review. Am J Public Health Nat Health 1962; 52: 925-32.

3.- Ferwerda A, Moll H, De Groot R. Respiratory tract infections by Mycoplasma pneumoniae in children: a review of diagnostic and therapeutic measures. Eur J Pediatr 2001; 160: 483-91.

4.- Broome C, Laventure M, Kaye H, Davis A, White H, Plikaytis B, et al. An explosive outbreak of Mycoplasma pneumoniae infection in a summer camp. Pediatrics 1980; 66; 884-8.

5.- Domínguez A, Minguell S, Torres J, Serrano
A, Vidal J, Salleras L. Community outbreak of acute respiratory infection by Mycoplasma pneumoniae. Eur J Epidemiol 1996; 12: 131-4.

6.- Centers for Disease Control and Prevention (CDC). Outbreaks of Mycoplasma pneumoniae respiratory infection: Ohio, Texas, and New York, 1993. MMWR Morb Mortal Wkly Rep 1993; 42: 931; 937-9.

7.- Layani-Milon M, Gras I, Valette M, Luciani J, Stagnara J, Aymard M, et al. Incidence of upper respiratory tract Mycoplasma pneumoniae infections among outpatients in Rhone-Alpes, France, during five successive winter periods. J Clin Microbiol 1999; 36: 1721-6.

8.- Harris J, Kolokathis A, Campbell M, Cassell G, Hammerschlag M. Safety and efficacy of azithromycin in the treatment of community-acquired pneumonia in children. Pediatr Infect Dis J 1998; 17: 865-71.

9.- $\quad$ Gendrel D, Raymond J, Moulin E, Iñíguez J, Ravilly S, Habib E, et al. Etiology and response to antibiotic therapy of community-acquired pneumonia in French children. Eur J Clin Microbiol Infect Dis 1997; 16: 388-91.

10.- Waites K. New concepts of Mycoplasma pneumoniae infections in children. Pediatr Pulmonol 2003; 36: 267-78.

11.- Michelow I, Olsen K, Lozano J, Rollins N, Duffy L, Ziegler T, et al. Epidemiology and clinical characteristics of community-acquired pneumonia in hospitalized children. Pediatrics 2004; 113: 701-7.

12.- Dorigo-Zetsma J, Wilbrink B, Van Del Nat H, Bartelds A, Heijnen Ml, Dankert J. Results of molecular detection of Mycoplasma pneumoniae 
among patients with acute respiratory infection and in their household contacts reveals children as human reservoirs. J Infect Dis 2001; 183: 675-8.

13.- Palma C, Martínez Ma, Salinas M, Rojas P. Portación faríngea de Mycoplasma pneumoniae en niños chilenos. Rev Chil Infect 2005; 22 : 247-50.

14.- Sánchez D. I, Cruz C, Kogan R, Lezana V, Martínez F, Pérz M A, Prado F, et al. Neumonía adquirida en la comunidad (sin inmunodeficiencia). Consenso de especialistas pediátricos, 1999. Rev Chil Enf Respir 1999; 15: 107-36.

15.- Murray H, Tuazon C. Atypical pneumonias. Med Clin North Am 1980; 64: 507-27.

16.- Cimolai N. Mycoplasma pneumoniae respiratory infection. Pediatr Rev 1998; 19: 327-31.

17.- Powell D. Mycoplasma pneumoniae. En: Behrman R, Kliegman R, Jenson H. Nelson: Tratado de Pediatría. Madrid, Editorial Elsevier, 2004. pp 990-2.

18.- Waites K, Talkington D. Mycoplasma pneumoniae and its role as a human pathogen. Clin Microbiol Rev 2004; 17: 697-728.

19.- Pickering L K, Baker C J, Long S S, McMillan J A. Mycoplasma pneumoniae Infections. En: Red Book: 2006 Report of the Committee on Infectious Diseases. 27a Ed. American Academy of Pediatrics, 2006. pp 468-70.

20.- Principi N, Esposito S. Emerging role of Mycoplasma pneumoniae and Chlamydia pneumoniae in paediatric respiratory-tract infections. Lancet Infect Dis 2001; 1: 334-44.

21.- Thibodeau K, Viera A. Atypical pathogens and challenges in community-acquired pneumonia. Am Fam Physician 2004; 69: 1699-706.

22.- Nagayama Y, Sakurai N, Yamamoto K, Honda A, Makuta M, Suzuki R. Isolation of Mycoplasma pneumoniae from children with lower-respiratory-tract infections. J Infect Dis 1988; 157: 911-7.

23.- Fernald G, Collier A, Clyde W. Respiratory infections due to Mycoplasma pneumoniae in infants and children. Pediatrics 1975; 55: 327 35.

24.- Ferrero F, Ossorio M, Eriksson P, Durán A. Mycoplasma pneumoniae en niños con neumonía. Arch Argent Pediatr 2000; 98: 12-7.

25.- Sáez-Llorens X, Castaño E, Wubbel L, Castrejon M, De Morales I, Vallarino D, et al. Importancia de Mycoplasma pneumoniae y Chlamydia pneumoniae en niños con neumonía adquirida en la comunidad. Rev Méd Panamá 1998; 23: 27-33.

26.- John S, Ramanathan J, Swischuk L. Spectrum of clinical and radiographic findings in pediatric mycoplasma pneumonia. Radiograph 2001; 21 : 121-31.

27.- Braun G, Wagner K, Huttner B, Schmid H. Mycoplasma pneumoniae: Usual suspect and unsecured diagnosis in the acute setting. $\mathrm{J}$ Emerg Med 2006; 30: 371-5.

28.- Othman N, Isaacs D, Kesson A. Mycoplasma pneumoniae infections in Australian children. J Paediatr Child Health 2005; 41: 671-6.

29.- Esposito S, Blasi F, Arosio C, Fioravanti L, Fagetti L, Droghetti, et al. Importance of acute Mycoplasma pneumoniae and Chlamydia pneumoniae infections in children with wheezing. Eur Respir J 2000; 16: 1142-6.

30.- Biscardi S, Lorrot M, Marc E, Moulin F, Boutonnat Faucher B, Heilbronner C, et al. Mycoplasma pneumoniae and asthma in children. Clin Infect Dis 2004; 38: 1341-6.

31.- Esposito S, Blasi F, Bellini F, Allegra L, Principi N. Mycoplasma pneumoniae and Chlamydia pneumoniae infections in children with pneumonia. Eur Respir J 2001; 17: 241-5.

32.- Principi N, Esposito S, Blasi F, Allegra L. Role of Mycoplasma pneumoniae and Chlamydia pneumoniae in children with communityacquired lower respiratory tract infections. Clin Infect Dis 2001; 32: 1281-9.
33.- Stevens D, Swift P, Johnston P, Kearney P, Corner B, Burman D. Mycoplasma pneumoniae infections in children. Arch Dis Child 1978; 53: 38-42.

34.- Chan E, Welsh C. Fulminant Mycoplasma pneumoniae pneumonia. West J Med 1995; 162 : $133-42$.

35.- Kaufman J, Cuvelier C, Van der Straeten M. Mycoplasma pneumoniae with fulminant evolution into diffuse interstitial fibrosis. Thorax 1980; 35: 140-4.

36.- Foy H, Nolan C, Allan I. Epidemiologic aspects of M. pneumoniae disease complications: a review. Yale J Biol Med 1983; 56: 469-73.

37.- Waris M, Toikka P, Saarinen T, Nikkari S, Meurman O, Vainiopaa R, et al. Diagnosis of Mycoplasma pneumoniae pneumonia in children. J Clin Microbiol 1998; 36: 3155-9.

38.- Souliou E, Almasri M, Papa A, Theodoridou A, Diza E. Laboratory diagnosis of Mycoplasma pneumoniae respiratory tract infections in children. Eur J Clin Microbiol Infect Dis 2007; 26: 513-5.

39.- Waites K, Taylor-Robinson D. Mycoplasma and Ureaplasma. Murray P, Baron E, Jorgensen J, Landry M, Pfaller M. Manual of Clinical Microbiology, $9^{\text {th }}$ edition, Washington DC, ASM Press, 2007, p. 1004-1020.

40.- Wahlgren H, Mortensson W, Eriksson M, Finkel Y, Forsgren M, Leinonen M. Radiological findings in children with acute pneumonia: age more important than infectious agent. Acta Radiol 2005; 46: 431-436.

41.- Shenoy V, Upadhyay S, Rao S, Shobha K. Mycoplasma pneumoniae infection in children with acute respiratory infection. J Trop Pediatr 2005; 51: 232-5.

42.- Reittner P, Muller N, Heyneman L, Johkoh T, Park J, Lee K, et al. Mycoplasma pneumoniae pneumonia: Radiographic and high-resolution ct features in 28 patients. Am J Radiol 2000; 174: $37-41$. 